\title{
Optical Properties of Micro-Spheres in Micro-Tube
}

\author{
S.E. Hao, H.Y. Li \\ Department of Chemistry \\ Harbin Institute of Technology \\ Harbin, China
}

\begin{abstract}
We proposed a fluorescent micro-sphere with diameter of $6 \mu \mathrm{m}$ was manipulated into a micro-tube with inner diameter of $6.2 \mu \mathrm{m}$. The Whispering Gallery Modes (WGMs) of fluorescence resonance were observed by $532 \mathrm{~nm}$ laser pumping the micro-spheres-micro-tube system. Another micro-sphere with the same diameter was manipulated into the micro-tube and mode splitting in the system of two spheres in contact in the micro-tube was demonstrated. We also discussed relationship between WGMs peak intensity and the excitation power. The scheme will bring new insight into the applications of WGMs for biomedical diagnostics and micro-fluidics.
\end{abstract} device

Keywords-WGM; Micro-tube; Micro-sphere; Integrated optical

\section{INTRODUCTION}

Owning to their high optical quality, long cavity lifetime and strong field confinement, optical whispering gallery modes (WGMs) micro-resonators have gained considerable attention over the last decades [1-4]. They have found their wide applications as optical filters [5], laser sources [6,7], and biological sensors [8, 9]. When light propagates circumferentially around the inside surface of a dielectric sphere, WGMs occur and confine photons in this small volume with a long time by total internal reflection. Coupled micro-spheres can be used as a well designed model system to learn spatial profiles of photonic molecule modes. For such a coupled structure the formation of a collective photon state is expected to appear as a mode splitting into a doublet [10]. In most experiments of measuring coherent coupling of WGMs $[11,12]$, the micro-spheres were dispersed on the substrate or in the water, and were manipulated into a line by a fiber probe or optical tweezers. In fact, if the bi-sphere are placed in the flowing gas or liquid, the measurement is difficult to accomplish. In this paper, we report on a fluorescent microsphere with diameter of $6 \mu \mathrm{m}$ was manipulated into a microtube with inner diameter of $6.2 \mu \mathrm{m}$. The WGMs were observed in the spectrum by pumping the micro-spheresmicro-tube system. By using spheres with nearly the same diameter, mode splitting in the system of two spheres in contact in the micro-tube was also demonstrated.

\section{EXPERIMENTAL SCHEMATIC}

Optical characterization of the micro-tube-micro-spheres system is carried out under an optical microscope, as schematically illustrated in Figure 1(a).

\author{
J. Li \\ College of Information Science and Engineering \\ Northeastern University \\ Shenyang, China
}

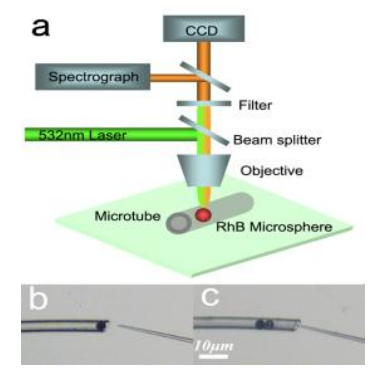

FIGURE I. .(A). THE SCHEMATIC EXPERIMENTAL CONFIGURATION. (B) ONE SINGLE MICRO-SPHERE WITH DIAMETER ABOUT $6 \mu \mathrm{M}$ IN THE MICRO-TUBE. (C) ANOTHER SPHERE WITH THE SAME DIAMETER WAS MANIPULATING INTO THE MICRO-TUBE.

The micro-tubes used in our experiment are fabricated by a flame-heated drawing method from the silica capillary tubing (outside diameter $\sim 162 \mu \mathrm{m}$, inner diameter $\sim 100 \mu \mathrm{m}$, refractive index 1.55, Poly micro Technologies, L. L. C.). We obtained silica micro-tubes with outside diameters down to $\sim 8$ $\mu \mathrm{m}$; wall thickness down to $\sim 900 \mathrm{~nm}$ and lengths up to hundreds of microns. A optical fiber probe with a top diameter of $\sim 1 \mu \mathrm{m}$ is fixed on a $x-y-z$ translation stage used to manipulate the micro-spheres into micro-tube. Figure 1(b) shows a single micro-sphere with diameter about $6 \mu \mathrm{m}$ was manipulating into the micro-tube by the probe. The inner diameter of the micro-tube is about $6.2 \mu \mathrm{m}$. We choose another sphere with the same size by measuring the diameter under microscope. Figure 1(c) shows another micro-sphere was pulled into the same micro-tube.

The tube is deposited on a low-index $\mathrm{MgF}_{2}$ substrate, a frequency doubled CW Q-switch Nd: YAG laser is focused to a spot size $\sim 100 \mu \mathrm{m}$ through a $40 \times$ objective $(\mathrm{NA}=0.45$ ) which allows for fluorescence from the spheres and laser power measurements at the same sample spot. The dye fluorescence is collected with the same objective, reflection of the $532 \mathrm{~nm}$ laser is removed by a filter, emission of the spheres is split by a beam splitter to a spectrometer (spectral resolution $=0.1 \mathrm{~nm}$ ) and CCD. The micro-tube-micro-spheres system is surrounded by air and all measurements are performed at room temperature.

\section{EXPERIMENTAL ANALYSIS AND DISCUSSION}

Figure 2(a) shows the spectra of the micro-tube-single sphere, at each step, an emission spectrum is collected with the rising distance of $1 \mu \mathrm{m}$. One can see that the resonance intensity of WGMs increases with the rising laser focus plane from P1 to P4 [Figure. 2(b)], and maximized at the junction of micro-spheres-surface with tube inner surface (P4). However, the resonance intensity of WGMs decreases sharply when the 
excitation and detection plane is positioned at the tube outside surface (P5), which mainly due to the transversal propagation in the tube and low coupling efficiency of the laser into the sphere. It is possible that the refractive index ratio between Melamine-Formaldehyde Resin sphere and silica capillary tubing $(1.68 / 1.55=1.08)$ is less, resulting in an inhomogeneous refractive index distribution on the microsphere surface and the WGMs are less well-confined inside the sphere, which should consequently broaden the modes resonances, as observed.

According to Abbe / Rayleigh resolution criterion, the resolution of the optical microscope is defined as the minimum distance between two same brightness of a point light can be distinguished by a microscope and it is following the formula: $\Delta x, \Delta y=\lambda / 2 n \sin \alpha=\lambda / 2 N A$ medium $\alpha$ is the semi-apre is the numerical aperture of the objective lens.) In the light propagation direction (z-axis), it is following the formula: $\Delta z=2 \lambda / n \sin ^{2} \alpha$

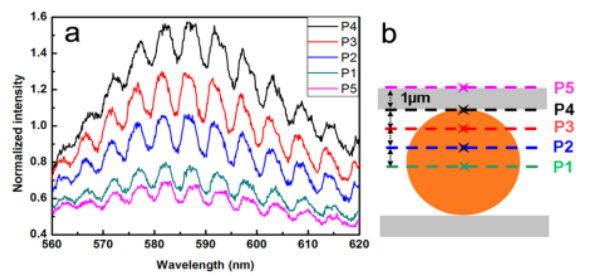

FIGURE II. SPECTRA OF THE SINGLE SPHERE IN THE TUBE WITH EXCITATION LASER FOCUS AT DIFFERENT PLANE OF THE SPHERE

(A). THE SCHEMES OF THE EXCITATION AND DETECTION AT DIFFERENT PLANE OF THE SPHERE (B). THE SPACING BETWEEN EACH TWO PLANE IS $1 \mu \mathrm{M}$.

We calculated the optical resolution of the $\mathrm{z}$-axis, $\Delta \mathrm{z} \approx 5.2$ $\mu \mathrm{m}$ in our experiment. However, this does not mean that light have the same energy density in the planes up and down of the focusing plane for $5 \mu \mathrm{m}$. To prove this, we focused a continuous wave laser $(\lambda=532 \mathrm{~nm})$ to a spot with diameter of $5 \mu \mathrm{m}$ on a MgF2 substrate by a $40 \times$ objective $(\mathrm{NA}=0.45)$ as shown in Figure.3.(1). We raised the MgF2 substrate up $1 \mu \mathrm{m}$ by adjusting the $x-y-z$ stage and obtained a dark-filed microscope image as shown in Figure.3.(1). Figure.3.(3-5) show the spot on the MgF2 substrate which was raised up 2 $\mu \mathrm{m}, 3 \mu \mathrm{m}$, and $4 \mu \mathrm{m}$, respectively. The increased spot size and decreased spot brightness are the evidence of the changing energy density in the up $5 \mu \mathrm{m}$ of the focusing plane. We also located one single micro-sphere with diameter of $5 \mu \mathrm{m}$ on the $\mathrm{MgF} 2$ substrate, then we raised the micro-sphere up $1 \mu \mathrm{m}$ each time to obtain corresponding microscope image as shown in Figure.3.(6-10). We can clearly see that the light focusing plane was dropped from the top of the micro-sphere to the bottom. Therefore, focusing laser on different cross-sections of the micro-sphere will result different excitation power.

To demonstrate the splitting of the WGMs in a structure composed of bi-sphere, we focus the excitation laser to the surface plane (P4) of the matched spheres and collect the emission spectra as shown in Figure 4. The inset images show dark-filed fluorescence microscope image of the single sphere (diameter $\sim 6 \mu \mathrm{m}$ ) and bi-sphere in contact in the tube. It is observed that spectra taken perpendicular to the axis of the bisphere (red line) show the characteristic mode split into a doublet compared with the single sphere (black line), which is due to the coherent coupling between the two matched sphere. 11,16 We will take the molecule mode detected at $\lambda=585.7$ $\mathrm{nm}$ for example, which is split to a red-shifted mode at 586.1 $\mathrm{nm}$ and a blue-shifted mode at $585.3 \mathrm{~nm}$. The measured wavelength splitting $\Delta \lambda$ of this mode is $0.8 \mathrm{~nm}$ which is close to the calculated $\Delta \lambda(\sim 0.53 \mathrm{~nm})$ according to Lorenz-Mie theory. In addition, the FWHM of the single sphere resonance peak at $\lambda=585.7 \mathrm{~nm}$ is $1.2 \mathrm{~nm}$, which is narrower than that of the bi-sphere in the tube $(\sim 1.7 \mathrm{~nm})$, corresponding $\mathrm{Q}$ factor $(\mathrm{Q}$ $=\lambda / \Delta \lambda$ ) of the single sphere is $\mathrm{Q} \approx 490$ and the bi-sphere is $\mathrm{Q}$ $\approx 340$. A $30 \%$ decrease in $Q$ value due to the additional loss mechanism caused by the strong inter-sphere coupling.

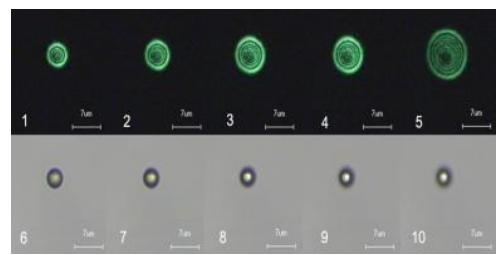

FIGURE III. DARK-FILED MICROSCOPE IMAGES OF THE FOCUSED SPOT (1-5). MICROSCOPE IMAGES OF A SINGLE MICRO-SPHERE WITH DIAMETER OF $5 \mu \mathrm{M}(6-10)$. THE SCALE BAR IN ALL IMAGES IS $7 \mu \mathrm{M}$.

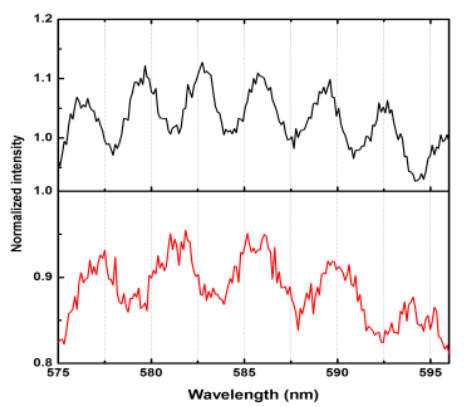

FIGURE IV. (COLOR ONLINE) THE SPECTRA OF THE MICRO-TUBESINGLE SPHERE (BLACK LINE) AND THE MICRO-TUBE-BI-SHPERE (RED LINE).

\section{Simulation RESUlts}

The electric field distribution in the micro-tube-singlesphere system and micro-tube-bi-sphere system are investigated with the three-dimensional finite-difference timedomain (3D-FDTD) simulations. A Software named OptiFDTD was used to simulate and calculate the electric field distribution. We select a cw $532 \mathrm{~nm}$ laser with power of 0.1 $\mathrm{mW}$ as the light source and the position of the light source was set in the border of the system. The light source is longer than the micro-tube as shown in Figure.5.(a). The results of the simulation is shown in Figure.5.(b), (c). It is noted that the electric field has been confined and distributed as circles around the center of the single sphere, while in the bi-sphere case, it has been horizontally distributed along the tube, which is further confirming that the strong coupling effect exists between the two spheres and the field strength weakened obviously. 


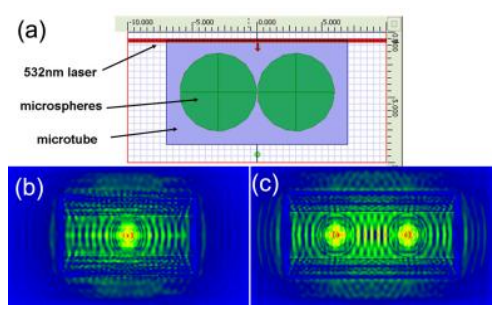

FIGURE V. (A) THE SCHEMATIC OF FDTD SIMULATION CONFIGURATION. (B) FDTD SIMULATION OF THE ELECTRIC FIELD INTENSITY DISTRIBUTION OF THE SINGLE MICRO-SPHERE AND THE BI-SPHERE IN THE TUBE (C).

\section{CONCLUSION}

In conclusion, we demonstrated WGMs could be excited and detected in dye-doped micro-spheres positioned in a micro-tube, and the intensity of WGMs changed with the excitation and detection plane from the center of the sphere to the outside surface of the tube. The resonance splitting was observed in the emission spectrum due to the strong coherent coupling between the two spheres, which permitted ample applications in micro-size optical sensors. In addition, the spheres covered by the micro-tube will allow the system placed in flowing gas or fluid. Therefore, this scheme will also bring new insight into the application of WGMs for biomedical diagnostics and micro-fluidics.

\section{ACKNOWLEDGMENT}

The authors thank the support from National Natural Science Foundation for Young Scholars of China under Grant 61405032 .

\section{REFERENCES}

[1] Benner, R.E., Barber, P.W., Owen, J.F. \& Chang, R.K., Observation of structure resonances in the fluorescence spectra from micro-spheres. Physical Review Letters, 44, pp.475, 1980.

[2] Spillane, S.M., Kippenberg, T.J. \& Vahala, K.J., Ultralow-threshold Raman laser using a spherical dielectric microcavity. Nature, 415, pp. 621-623, 2002.

[3] Vahala, K.J., Optical microcavities. Nature, 424, pp. 839-846, 2003.

[4] Gérard D., Devilez A., Aouani H., Stout B., Bonod N., Wenger J., Popov E. \& Rigneault, H., Efficient excitation and collection of singlemolecule fluorescence close to a dielectric micro-sphere. Journal of the Optical Society of America B, 26, pp. 1473-1478, 2009.

[5] Boriskina, S.V., Benson, T.M., Sewell P. \& Nosich, A.I., Tuning of Elliptic Whispering-gallery-mode microdisk waveguide filters. Journal of Lightwave Technology, 21, pp. 1987, 2003.

[6] Sandoghdar, V., Treussart, F., Hare, J., Lefèvre-Seguin, V., Raimond, J. M. \& Haroche, S., Very low threshold whispering-gallery-mode microsphere laser. Physical Review A, 54, pp. 1777, 1996.

[7] Lee, S.B., Oh, M.K., Lee, J.H. \& An, K., Single radial-mode lasing in a submicron-thickness spherical shell microlase. Appllied Physics Letters, 90, pp. 201102, 2007.

[8] Francois, A. \& Himmelhaus, M., Whispering gallery mode biosensor operated in the stimulated emission regime. Appllied Physics Letters, 94, pp. 031101, 2009.

[9] Vollmer, F., Braun, D., Libchaber, A., Khoshsima, M., Teraoka, I. \& Arnold, S., Protein detection by optical shift of a resonant microcavity. Appllied Physics Letters, 80, pp. 4057-4059, 2002.

[10] Li, H.Y., Hao, S.E., Qiang, L.S., Li, J., \& Zhang, Y.D., Observation of whispering gallery modes in micro-tube-microspheres system. Appllied Physics Letters, 102, pp. 231908, 2013.
[11] Mukaiyama, T., Takeda, K., Miyazaki, H., Jimba, Y., \& KuwataGonokami, M., Tight-binding photonic molecule modes of resonant bispheres. Physical Review Letters, 82, pp. 4623, 1999.

[12] Thompson, D.B., Keating, D.A., Guler, E., Ichimura, K., Williams, M. E. \& Fuller, K.A., Separation-sensitive measurements of morphology dependent resonances in coupled fluorescent micro-spheres. Optics Express 18, pp. 19209-19218, 2010. 